\title{
Identification of ZNF704 as a Novel Oncogene and an Independent Prognostic Marker in Chondrosarcoma
}

\author{
Changbao Chen' \\ Hua Zhou ${ }^{2}$ \\ Xiaolin Zhang' \\ Zhongjun Liu $^{2}$ \\ Xinlong $\mathrm{Ma}^{\mathrm{I}}$ \\ 'Department of Spinal Surgery, Tianjin \\ Hospital, Tianjin, 3002II, People's \\ Republic of China; ${ }^{2}$ Department of \\ Orthopaedic Surgery, Peking University \\ Third Hospital, Beijing, I0019I, People's \\ Republic of China
}

Purpose: The transcription factor zinc finger protein 704 (ZNF704) is implicated in tumorigenesis. However, the underlying role of ZNF704 in the pathogenesis of chondrosarcoma remains not well delineated. This study investigates the expression level, prognostic significance and potential biological function of ZNF704 in human chondrosarcoma.

Materials and Methods: The mRNA and protein levels of ZNF704 in fresh chondrosarcomas and the paired adjacent non-tumor tissues were evaluated using real-time PCR and immunoblotting, respectively. The protein expression of ZNF704 in chondrosarcoma specimens was detected by immunohistochemistry, and the associations among its expression level, clinicopathological characteristics and prognosis were further investigated. Cell viability, colony formation and apoptosis assay were determined in chondrosarcoma cells and a xenograft model with ZNF704 knockdown.

Results: The expression levels of ZNF704 mRNA and protein in chondrosarcoma tissues were significantly higher than those in the paired adjacent non-tumor tissues and benign cartilage tumors. Clinicopathological analysis revealed that ZNF704 was expressed at higher levels in chondrosarcoma patients with higher histological grade and advanced MSTS stage. We also found that high expression of ZNF704 significantly correlated with a worse overall survival of chondrosarcoma patients. Multivariate Cox regression analysis indicated that ZNF704 was an independent prognostic marker in chondrosarcoma patients. Our in vitro studies demonstrated that knockdown of ZNF704 markedly inhibited chondrosarcoma cell viability, colony formation and induced apoptosis. In a nude mouse xenograft model, ZNF704 knockdown slowed down chondrosarcoma growth by inducing apoptosis in vivo.

Conclusion: These findings suggest that ZNF704 may act as a potent oncogene implicated in chondrosarcoma development, and serve as a independent prognostic marker, highlight the potential of ZNF704 as a novel biomarker and therapeutic target for chondrosarcoma.

Keywords: chondrosarcoma development, ZNF704, cell growth, prognostic biomarker, therapeutic target

\section{Introduction}

Chondrosarcoma (CS) is a heterogeneous group of slow-growing primary cartilageforming malignancy, characterized by the formation of neoplastic hyaline cartilaginous tissues. Conventional chondrosarcoma, as the vast majority of chondrosarcomas, poorly responds to chemotherapy and radiation treatment, which results in high morbidity and mortality rates. ${ }^{1}$ Recent discoveries in the biology, genetics and epigenetics of chondrosarcoma have significantly advanced our understanding of the pathobiology of chondrosarcoma, which may offer insight into
Correspondence: Xinlong Ma Department of Spinal Surgery, Tianjin Hospital, 406 Jiefang South Road, Tianjin, 3002 I I, People's Republic of China

Email richivaldchen@gmail.com 
potential therapeutic targets. ${ }^{2}$ Thus, novel targeted therapies based upon the molecular mechanisms of chondrosarcoma development, representing a novel relevant therapeutic approach, will open new treatment options by targeting several signaling pathways responsible for the biological processes such as proliferation, differentiation and apoptosis. ${ }^{3}$

The zinc finger protein family, as a class of DNAbinding transcription factors, plays a pivotal role in regulating development, differentiation, metabolism and apoptosis, and also implicated in tumorigenesis. ${ }^{4}$ As a general transcriptional factor, zinc finger protein 704 (ZNF704), mapped to 8q21, specifically binds to DNA that activates the expression of protein-coding and noncoding genes, and is frequently amplified in various cancers and participates in numerous cellular biological activities. $^{5,6}$ To date, little is known about the biological functions of ZNF704, although one study indicates that it plays a role in malignant transformation. ${ }^{7}$ Recent reports have revealed that ZNF704 is frequently increased in brease and prostate cancers, ${ }^{6,8}$ exerting an oncogenic role in carcinogenesis. However, data from the Cancer Genome Atlas showed that lower expression of ZNF704 was detected in prostate tumors as compared to benign prostate samples. ${ }^{8}$ Therefore, further work will be needed to understand the underlying role of ZNF704 in carcinogenesis.

To address the potential role of ZNF704 in chondrosarcoma, we initially detected the expression profile and clinical value of ZNF704, and further explored its biological functions in chondrosarcoma development. Our findings demonstrated that ZNF704 expression is remarkably overexpressed in chondrosarcoma specimens and significantly correlated with high-grade chondrosarcoma and poor prognosis. Moreover, ZNF704 is recognized as an independent prognostic factor for the overall survival of chondrosarcoma patients. Knockdown of ZNF704 significantly inhibits chondrosarcoma cell growth through induction of apoptosis in vitro and in vivo. These findings highlight the potential of ZNF704 as a novel biomarker and therapeutic target for chondrosarcoma.

\section{Materials and Methods}

\section{Clinical Specimens and Cell Line}

Clinical specimens such as fresh chondrosarcoma tissues, the paired adjacent non-tumor tissues, conventional chondrosarcoma and benign cartilage tumors were enrolled as described previously. ${ }^{9}$ Clinical clinicopathological information and follow-up data were reported previously, and signed informed consent for sample collection and analysis were obtained from all patients and was in full compliance with national legislation and the ethical standards as described previously (IRB00001052-08044). ${ }^{10,11}$ Human chondrosarcoma SW1353 cell line was purchased from the American Type Culture Collection (Bethesda, MD, USA) and maintained in a humidified cell incubator with $5 \% \mathrm{CO}_{2}$ at $37^{\circ} \mathrm{C}$.

\section{RNA Extraction and Real-Time PCR}

Total RNA extraction, cDNA synthesis, and qRT-PCR were performed as described previously. ${ }^{10}$ Typically, the primer pairs used for ZNF704 were followed as Forward: 5'- GATCAAGCTCAACACAGACTCA-3'; Reverse: 5'TCTGGGATGGGGAAAGTAGGA-3' as described previously. ${ }^{6}$ The data were shown as $2^{-\Delta \Delta \mathrm{Ct}}$ values and were representative of at least three independent experiments.

\section{Protein Extraction and Western Blot Analysis}

Protein extraction and Western blot were performed as previously described. ${ }^{12}$ Primary antibodies against primary ZNF704 (Abcam, ab121804) and $\beta$-actin (Sigma-Aldrich, A5316) were used at 1:1000 dilution. An HRP-conjugated anti-rabbit IgG or anti-mouse IgG (Sigma) was used at a 1:5000 dilution and detected using Immobilon Western Chemi-luminescent HRP Substrate (Millipore). ${ }^{12}$

\section{Immunohistochemistry and Evaluation of Staining}

Detailed experimental protocols have been described previously. ${ }^{10,11}$ Typically, after antigen retrieval, ZNF704 (Abcam, ab121804; 1:100) immunoreactivities were detected using the EnVision+, Peroxidase system (DAKO Diagnostics, Denmark). The slides were incubated with DAB substrate (DAKO Diagnostics, Denmark) and counterstained with hematoxylin. The percentage of positive tumor cells was graded as per the following criteria: 0 , less than $10 \% ; 1,10-25 \% ; 2,26-50 \% ; 3$, more than $50 \%$. Nucleus immunoreactivity of ZNF704 was assessed as follows: (1) a low expression level, recognized as scores of 0 for no staining and $1+$ for weak staining; and (2) a high expression level, recognized as a score of $2+$ for moderate staining and $3+$ for strong staining. ZNF704 
nucleus immunoreactivity was considered as either low (score $0-1$ ) or high (scores 2 to 3 ).

\section{Generation of Stable Cell Clones with ZNF704 Knockdown}

Stable SW1353 cell clones with ZNF704 knockdown were generated using SMARTvector Lentiviral shRNA targeting ZNF704 (shZNF704) and a negative control vector (shCont) from Dharmacon. To select stable transfectants, the SW1353 cells were transfected and incubated overnight, and then switched to a medium containing $600 \mu \mathrm{g} /$ $\mathrm{mL}$ G418 for further incubation, and a pool of G418resistant cells was selected for further studies as per the manufacturer's instructions. ${ }^{10}$

\section{Cell Viability and Clonogenic Assays}

Cell Counting Kit-8 (Dojindo, Kumamoto, Japan) for cell viability, and clonogenic formation assays were conducted as described previously. ${ }^{10}$ Briefly, SW1353 cells were seeded into 96-well plates at 5000 cells per well for 24 hours and assessed using CCK-8 at the indicated time. For clonogenic assay, after treatment, the number of viable cell colonies was determined after either 10 days SW1353 cells post inoculation of 150 cells per well in triplicate in 12well plates. Colonies were counted after methanol/acetone (1:1) fixation and Gentian Violet staining according to the previous experimental protocols. ${ }^{11}$

\section{Detection of Apoptosis and Caspase-3 Activity Assay in vitro}

After treatment, cells were resuspended in $100 \mu \mathrm{L}$ of binding buffer, and immediately analyzed on a FACS Calibur flow cytometer (Becton Dickinson, USA) with Cell Quest Software (BD bioscience, USA) to calculate apoptotic cells. The caspase-3 activity was evaluated using a Caspase-3/CPP32 Fluorometric Assay Kit (Biovision, USA) according to the manufacturer's instructions, as described in our previous study. ${ }^{12}$

\section{TUNEL Assay in vivo}

To detect the apoptosis index in xenograft model in vivo, TUNEL (terminal-deoxynucleotidyl transferase mediated nick end labeling) assay was conducted by an In Situ Cell Death Detection kit, POD (Roche Diagnostics, USA) to identify apoptotic cells present in tumor specimens according to the manufacturer's directions. The percentage of DAB-stained apoptotic cells was calculated as the mean of ten different fields from each specimen as described in our previous study. ${ }^{12}$

\section{Chondrosarcoma Xenograft Model}

Chondrosarcoma xenograft model in nude mice was established according to our previous study. ${ }^{12}$ Mice were housed and maintained in a pathogen-free facility, and all experimental procedures and protocols of the animal study were approved by the Animal Care and Use Committee and strictly followed ethical regulatory standards. Briefly, stable SW1353 cell clones with shCont (Control) or shZNF704 were collected and resuspended at a density of $5 \times 10^{6}$ cells per $100 \mu \mathrm{L}$. BALB/c nude mice were subcutaneously implanted into the right flank. The tumors were measured with digital calipers at indicated time points for 21 days post inoculation. The tumor volume was calculated with the formula $\mathrm{V}=\mathrm{L} \times \mathrm{W}^{2} \times 1 / 2(\mathrm{~V}$, volume; $\mathrm{L}$, length; $\mathrm{W}$, width of tumor). The excised tumors were weighed and further analyzed for the TUNEL assay.

\section{Statistical Analysis}

All data are presented as the Mean \pm SD. The Student's $t$-test or the Pearson's Chi-square test/Fisher's exact test was utilized to analyze difference between groups with continuous variables or categorical variables, respectively. The Kaplan-Meier method and the Log rank test were applied for overall survival with chondrosarcoma patients. Multivariate analyses of clinicopathological factors, ZNF704 level and overall survival were performed using Cox regression model. Statistical analyses were performed using SPSS (SPSS, Chicago, IL, USA.) and $P<0.05$ was considered to be statistically significant. All reported $P$ values were two-sided.

\section{Results}

\section{Increased Levels of ZNF704 in}

\section{Chondrosarcoma Specimens}

To address the potential role of ZNF704 in chondrosarcoma, we evaluated the ZNF704 expression levels in 6 paired cases of fresh chondrosarcoma tissues and the paired adjacent non-tumor tissues using Real-time PCR and immunoblotting, respectively. As shown in Figure 1A, the mRNA expression level of ZNF704 was significantly higher in chondrosarcoma tissues than that in the adjacent non-tumor tissues $(P<0.01)$. Furthermore, we performed immunoblotting to examine the protein levels of ZNF704, and quantitative analysis showed that the 


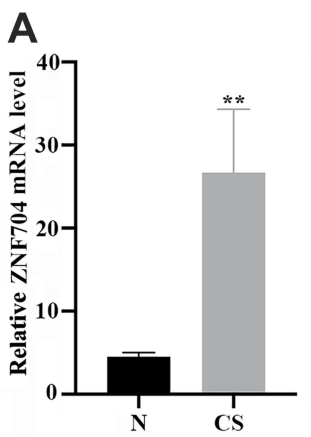

B
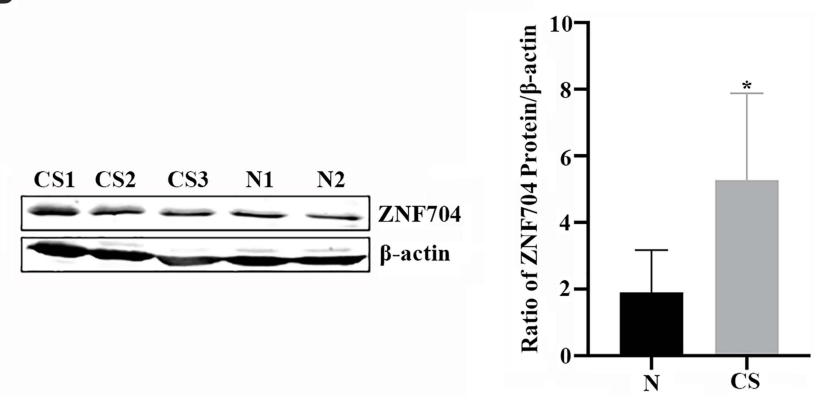

Figure I The expression level of zinc finger protein 704 (ZNF704) in fresh chondrosarcoma tissues and the paired adjacent non-tumor tissues. (A) Comparison of differences in the ZNF704 mRNA levels between 6 cases of chondrosarcoma tissues and the paired adjacent non-tumor tissues. CS, chondrosarcoma tissues; N, the paired adjacent non-tumor tissues. $* * P<0.01$ by Student's $t$ test. (B) Representative immunoblotting showed the protein expression of ZNF704 in CS tissues and the paired adjacent non-tumor tissues (Left Panel). Quantitative data revealed that the ZNF704 protein expression in CS tissues was significantly higher than that in the paired adjacent nontumor tissues (Right Panel). $* P<0.05$ by Student's $t$ test.

protein level of ZNF704 in chondrosarcoma tissues was also higher compared with that in the adjacent non-tumor tissues $(P<0.05$, Figure $1 \mathrm{~B}$ and $\mathrm{C})$.

To further investigate the expression profile of ZNF704 in human cartilage-forming tumors, we tested the ZNF704 protein expression using immunohistochemistry assay in a retrospective cohort of 63 cases of conventional chondrosarcomas and 17 cases of benign cartilage tumors, and revealed that the positive signal of ZNF704 was preferentially recognized at the nucleus in human cartilage tumors such as osteochondroma and chondrosarcoma (Figure 2A and B). In these 63 chondrosarcoma cases, high expression of ZNF704 was detected in 39 (61.9\%) cases of chondrosarcomas, whereas only $5(29.4 \%)$ cases of benign cartilage tumors showed a high ZNF704 expression $(P=0.027$, Table 1), suggesting that increased levels of ZNF704 may be implicated in the pathogenesis of human chondrosarcoma.

\section{Clinical Significance of ZNF704 in Patients with Chondrosarcoma}

As shown in Table 2, clinical association analysis delineated that increased level of ZNF704 protein expression in chondrosarcoma tissues was prominently correlated with severely poor clinicopathologic features including higher histological grade $(P=0.006)$ and advanced MSTS stage $(P=0.002)$. Thus, our results indicated that the protein expression of ZNF704 was remarkably increased in chondrosarcoma tissues, which was significantly associated with poor prognostic features.

Next, we determined whether ZNF704 expression status may have clinical prognostic value on 63 patients with chondrosarcoma. We had constructed Kaplan-Meier survival curves using the overall survival date to analyze cases with high or low ZNF704 level. As shown in Figure $2 \mathrm{C}$, the median overall survival times in the high and low ZNF704 subgroups of chondrosarcoma patients were 51.7 months and 86.0 months, respectively (Figure 2C). Patients with high ZNF704 expression group $(n=39)$ indeed had a significantly poorer prognosis than those with low ZNF704 expression group $(\mathrm{n}=24)$ (log-rank=7.342; $P=0.007$; Figure 2C). Moreover, to identify the independently prognostic factors for the overall survival of chondrosarcoma patients, we had performed multivariate Cox regression model analysis, and found that high ZNF704 level was recognized as an independent prognostic factor for poor overall survival of chondrosarcoma patients (HR, 7.159, $P=0.005$, Table 3 ). These data indicate that ZNF704 may act as a potent biomarker for predicting prognosis of chondrosarcoma patients.

\section{ZNF704 Knockdown Inhibits Tumor Growth and Induces Apoptosis in vitro and in vivo}

A previous study has demonstrated that ZNF704 could be a potential oncogenic factor, by disrupting circadian rhythm of breast cancer cells and contributing to breast carcinogenesis. ${ }^{6}$ To identify the role of ZNF704 in chondrosarcoma, we prepared shRNA oligonucleotide specifically targeting ZNF704 expression (shZNF704). As measured by immunoblotting analysis, ZNF704 protein levels were significantly reduced by shZNF704 as compared to that of shRNA control (shCont) in chondrosarcoma SW1353 cells (Figure 3A). Stable transfected cell 
A
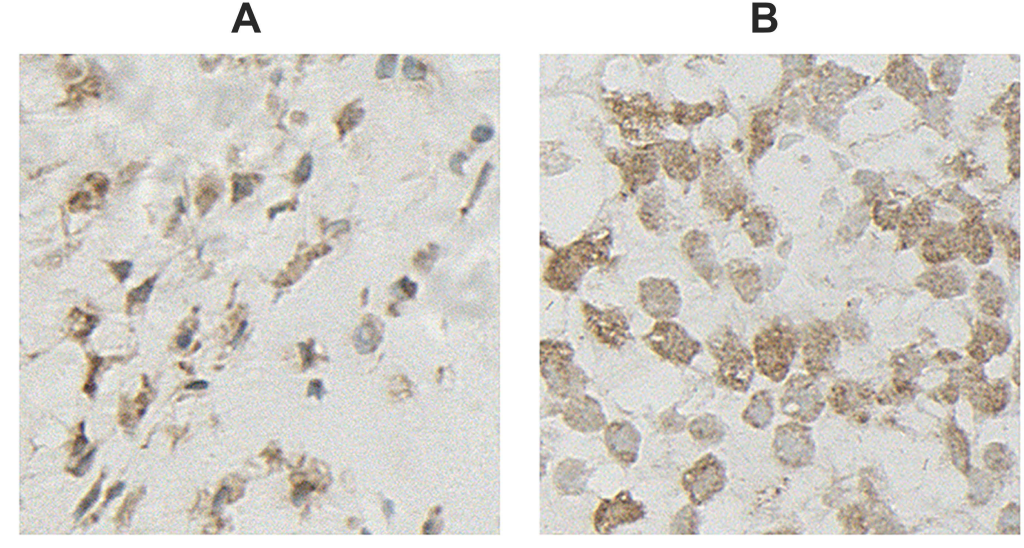

C

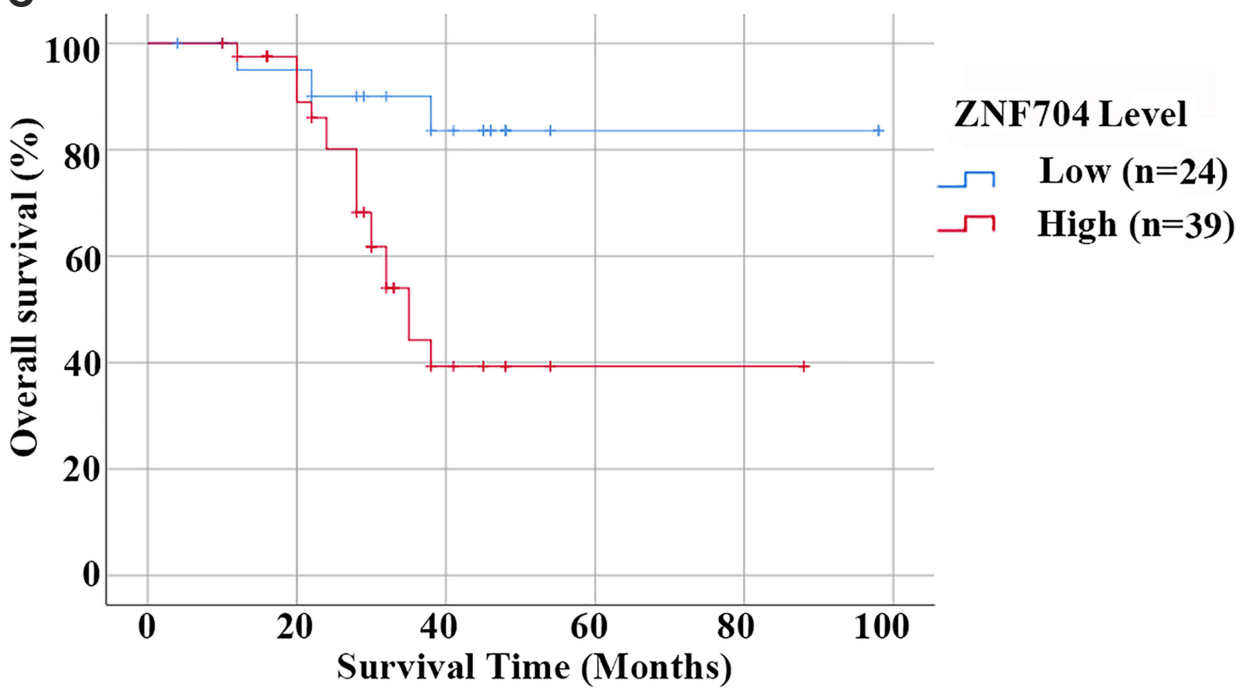

Figure 2 Prognostic value of ZNF704 in chondrosarcoma cases. (A and B) Representative immunostaining of ZNF704 were shown in human cartilage tumors. Osteochondroma with low nucleus immunostaining of ZNF704 (A). High (B) nucleus immunostaining of ZNF704 were detected in chondrosarcoma tissues. (C) Prognostic significance of ZNF704 in 63 patients with chondrosarcoma. Kaplan-Meier overall survival curves and the Log rank test of 63 cases of chondrosarcoma patients based upon the status of ZNF704 expression were conducted, and unravelled that high ZNF704 expression remarkably correlated with poor prognosis $(P=0.01)$.

clones were collected and subjected to CCK-8, clonogenic and flow cytometry assays to evaluate cell proliferation and apoptosis, respectively. As shown in Figure 3B and C, ZNF704 knockdown significantly inhibited SW1353 cell viability and colony formation for both assays $(P<0.05)$. Furthermore, as determined by flow cytometry, the

Table I The Protein Expression Level of ZNF704 in Human Cartilage Tumors

\begin{tabular}{|l|c|c|c|c|}
\hline Groups & $\begin{array}{c}\text { Total No. of } \\
\text { Cases }\end{array}$ & \multicolumn{2}{|c|}{$\begin{array}{c}\text { ZNF704 } \\
\text { Expression }\end{array}$} & \multirow{2}{*}{ P-value } \\
\cline { 2 - 4 } & & Low & High & \\
\hline Benign cartilage tumor & 17 & 12 & 5 & $0.027^{*}$ \\
Chondrosarcoma & 63 & 24 & 39 & \\
\hline
\end{tabular}

Notes: $P$ values recorded are the results from Fisher's Exact Tests. $* P<0.05$. percentage of apoptotic SW1353 cells were significantly elevated after ZNF704 knockdown $(P<0.01$, Figure 3D). In addition, our data have unravelled that ZNF704 knockdown definitely triggered the increased activity of caspase $3(P<0.01$, Figure 3E).

Subsequently, we implanted stable SW1353 cell clones with shZNF704 or shCont into nude mice via subcutaneous injection to examine whether ZNF704 knockdown influenced tumor growth in mice. As shown in Figure $3 \mathrm{~F}$ and $\mathrm{G}$, tumor growth curves and tumor weight revealed that ZNF704 knockdown significantly slowed down tumor growth in mice $(P<0.05)$. We also detect apoptosis index in these xenograft tissues using TUNEL assays, and found that consistent with our in vitro data, ZNF704 knockdown significantly induced apoptosis in vivo $(P<0.01$, Figure $3 \mathrm{H})$. Thus, ZNF704 
Table 2 Associations Between ZNF704 Expression and the Clinicopathological Characteristics in 63 Patients with Chondrosarcoma

\begin{tabular}{|c|c|c|c|c|}
\hline \multirow[t]{2}{*}{$\begin{array}{l}\text { Clinicopathological } \\
\text { Factors }\end{array}$} & \multirow[t]{2}{*}{$\begin{array}{c}\text { Total No. of } \\
\text { Cases }\end{array}$} & \multicolumn{2}{|c|}{$\begin{array}{c}\text { ZNF395 } \\
\text { Expression }\end{array}$} & \multirow[t]{2}{*}{$P$-value } \\
\hline & & Low & High & \\
\hline Gender & & & & 0.298 \\
\hline Male & 36 & 16 & 20 & \\
\hline Female & 27 & 8 & 19 & \\
\hline Age (years) & & & & 0.759 \\
\hline$\geq 40$ & 49 & 18 & 31 & \\
\hline$<40$ & 14 & 6 & 8 & \\
\hline Anatomical Location & & & & 0.410 \\
\hline Limb bone & 21 & 6 & 15 & \\
\hline Axial bone & 42 & 18 & 24 & \\
\hline Histological Grade & & & & $0.006^{*}$ \\
\hline Well-differentiated (I) & 32 & 18 & 14 & \\
\hline Moderately (II) & 19 & 5 & 14 & \\
\hline Poorly (III) & 12 & I & 11 & \\
\hline MSTS stage & & & & $0.002 *$ \\
\hline$I A+I B$ & 34 & 19 & 15 & \\
\hline$\|A+\| B$ & 29 & 5 & 24 & \\
\hline
\end{tabular}

Notes: $P$ values recorded are the results from Chi-square Tests or Fisher's Exact Tests. ${ }^{*} P<0.05$. MSTS stage represents Musculoskeletal Tumor Society stage.

knockdown exerts an anti-chondrosarcoma effect by suppressing cell proliferation and inducing apoptosis, suggesting ZNF704 can be a novel oncogene implicated in the pathogenesis of chondrosarcoma.

\section{Discussion}

Zinc finger proteins as DNA-binding transcription factors function in the biological processes such as development, differentiation, proliferation and apoptosis. ${ }^{5}$ ZNF704, as a transcriptional factor, plays a role in malignant transformation, ${ }^{7}$ and is overexpressed in breast cancer and may be a potential oncogenic factor by disrupting circadian rhythm of breast cancer cells and contributing to breast carcinogenesis. ${ }^{6}$ Although data from the Cancer Genome Atlas showed that lower expression was detected in prostate cancers as compared to benign prostate samples, they definitely found higher ZNF704 expression in patients with metastatic progression. ${ }^{8}$ Thus, further work will be needed to understand the role of ZNF704 in aggressive prostate cancers. Together, these findings had implied that ZNF704 may exert a potent oncogenic role in potentiating carcinogenesis. In deed, little is known about the underlying biological role of ZNF704 in carcinogenesis. In regard to chondrosarcoma, the potential role of ZNF704 in the pathogenesis of chondrosarcoma remains elusive. In this study, we first demonstrated that ZNF704 was significantly increased in chondrosarcoma tissues as compared to the paired adjacent non-tumor tissues and benign cartilage tumors. Our findings supported the notion that ZNF704 might exert a oncogenic role in chondrosarcoma development in vivo, representing a novel mechanism for dysregulation of ZNF704 linked to the pathogenesis of chondrosarcoma.

One study has documented that tumor marker ZNF704 as part of a four gene transcript score has prognostic value for metastatic-lethal progression in prostate cancer, ${ }^{8}$ suggesting it could be a prognostic potential. To characterize the clinical significance of ZNF704 in chondrosarcoma, we presented the first evidence that high expression of ZNF704 was significantly correlated with higher histological grade and advanced MSTS stage, rather than age, gender and anatomical location (Table 2). Importantly, our data shown that high ZNF704 level had significantly unfavourable impact on the overall survival of chondrosarcoma patients (Figure 2C), which was consistent with the previous studies. ${ }^{8}$ Furthermore, multivariate Cox regression model analysis found that ZNF704 was identified as an

Table 3 Multivariate Analysis of Various Prognostic Parameters in 63 Patients with Chondrosarcoma by the Cox Regression Model

\begin{tabular}{|l|c|c|c|c|}
\hline \multirow{2}{*}{ Variables } & Unfavorable vs Favorable & \multicolumn{3}{|c|}{ Multivariate Analysis } \\
\cline { 2 - 5 } & & Hazard Ratio & 95\% Confidence Interval & P value \\
\hline Gender & Male vs Female & 2.916 & $0.983-8.646$ & 0.054 \\
Age & $\geq 40$ vs <40 & 1.08 I & $0.283-4.130$ & 0.909 \\
Anatomical location & Axial vs Limb bone & 2.695 & $0.817-8.894$ & 0.104 \\
Histological grade & Grade 3 vs Grade I and 2 & 3.244 & $1.165-9.027$ & $0.024^{*}$ \\
MSTS stage & II A + II B vs IA + I B & 4.636 & $1.574-13.658$ & $0.005^{*}$ \\
ZNF704 level & High vs Low & 7.159 & $1.814-28.244$ & $0.005^{*}$ \\
\hline
\end{tabular}

Notes: $P$ values recorded are the results from Cox Regression Analysis. ${ }^{*} P<0.05$ 
A

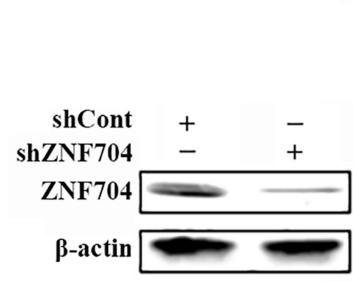

C

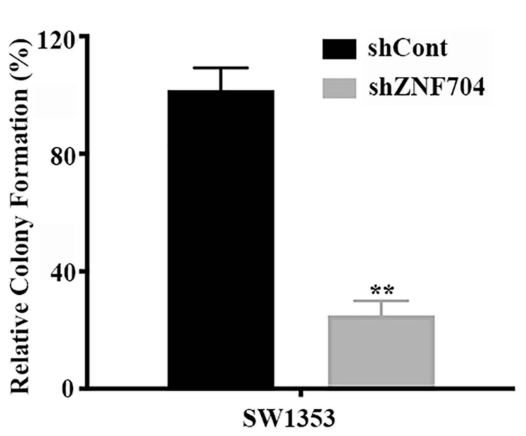

F

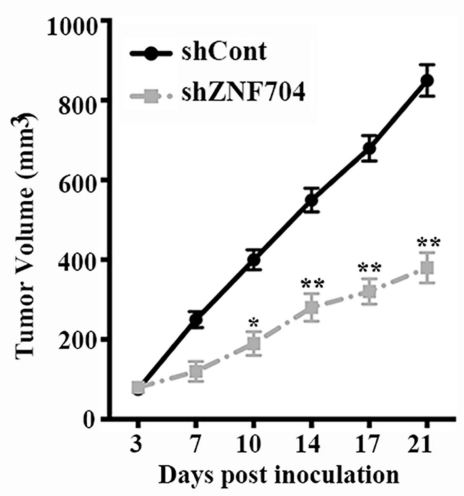

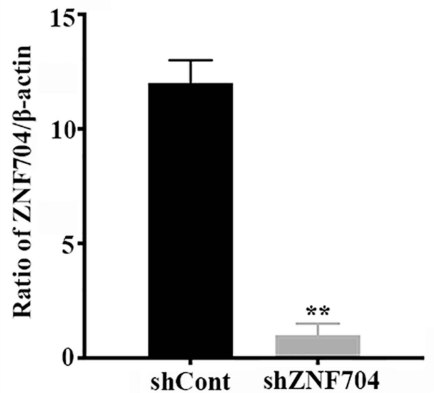

D

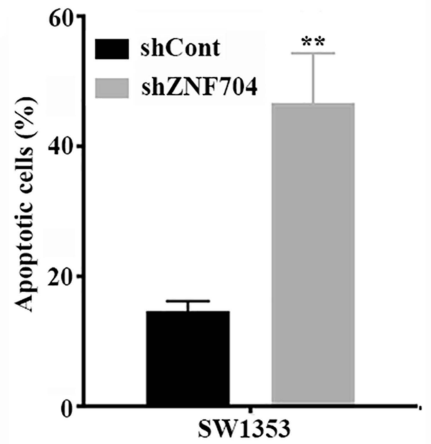

G

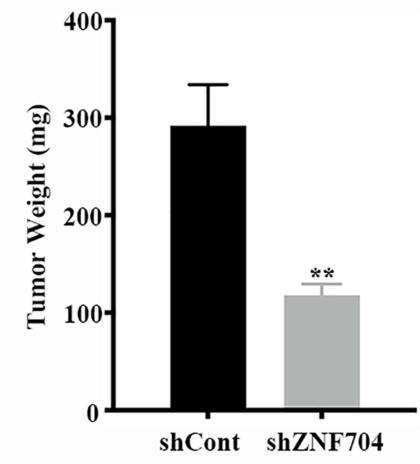

B

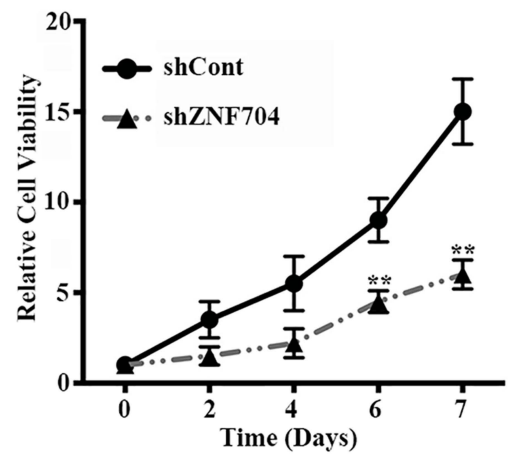

E

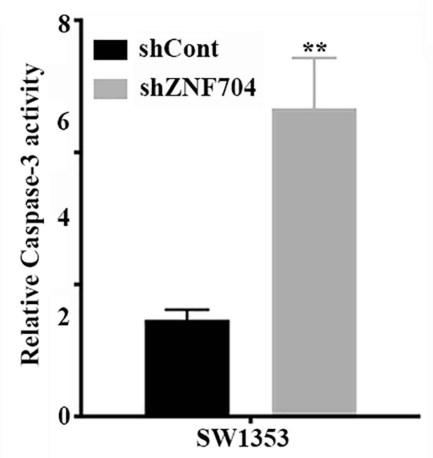

H

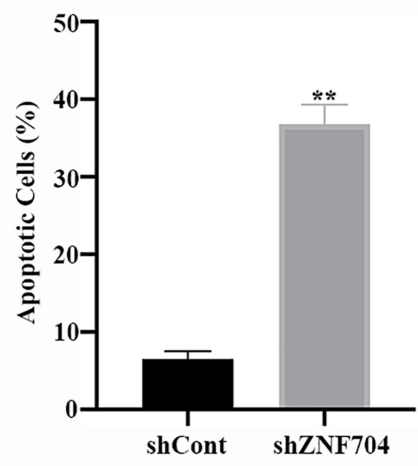

Figure 3 Knockdown of ZNF704 inhibits chondrosarcoma cell proliferation and induces apoptosis in vitro and in vivo. (A) ZNF704 was markedly reduced by shRNA against ZNF704. SWI353 cells transfected with control shRNA (shCont) or shRNA against ZNF704 (shZNF704) were subjected to immunoblotting (Left Panel). The histograms shown the gray intensity analysis to the bands of immunoblotting (Right Panel, **P <0.0I). (B and C) The inhibitory effects of shRNA against ZNF704 on cell viability and colony formation. Stable SWI353 cell clones with shCont or shZNF704 were incubated for the indicated time, and then cell viability (B) and colony formation (C) were detected by CCK-8 and clonogenic assay respectively, as indicated in Material and Methods. Data are plotted as the mean \pm SD (*P $<0.05$, $* * P<0.0$ I). (D) $Q$ Quantification of the apoptotic cell population by flow cytometry. Stable SWI353 cell clones with shCont or shZNF704 were incubated for 48 hours. Apoptotic cells were measured by FITC-Annexin V and PI staining followed by flow cytometry analysis. Knockdown of ZNF704 induced a larger subset of apoptotic cells compared with shCont. $\mathrm{n}=3$ repeats with similar results; ${ }^{* * P}<0.01$ by Student's $t$ test. (E) Quantification of the pro-apoptotic caspase 3 activity. Stable SWI 353 cell clones with shCont or shZNF704 were incubated for 48 hours. Caspase- 3 activity was measured and revealed that the pro-apoptotic caspase 3 activity was significantly increased after knockdown of ZNF704. $n=3$ repeats with similar results. **P $<0.0$ I by Student's $t$ test. ( $\mathbf{F}$ and $\mathbf{G})$ Knockdown of ZNF704 suppressed tumor growth in a nude mouse xenograft model. Stable SWI 353 cell clone with shCont or shZNF704 were implanted into nude mice via subcutaneous injection. Tumor volume (F) and tumor weight (G) of stable SWI353 cell clones for 21 days, respectively, indicated that ZNF704 knockdown slowed down tumor growth in nude mice. $n=5$, Data are plotted as the mean \pm standard deviation $(* P<0.05$, $* * P<0.0 \mathrm{I})$. (H) Quantification of apoptotic cells using TUNEL assay in SWI353 chondrosarcoma xenografts. The average percentage of TUNEL-positive cells in tumors isolated from shZNF704 were significantly higher than those in the shCont group. $n=5, * * P<0.01$.

independent factor in predicting the overall survival in chondrosarcoma patients (Table 3), suggesting that ZNF704 can be identified as an independent prognostic biomarker for chondrosarcoma patients, in line with a potential biomarker in prostate cancer. ${ }^{8}$ In agreement with the previous reports that high expression of ZNF704 
had a markedly poorer prognosis, ${ }^{8}$ our results shown that the status of ZNF704 is critical for prognosis determination in chondrosarcoma patients. Thus, our findings might help the clinician to determine the prognosis of chondrosarcoma based upon the status of ZNF704, suggesting the potential of ZNF704 as a novel biomarker in chondrosarcoma patients. However, further investigations for the clinical value of ZNF704 in larger cohorts of chondrosarcomas are indeed needed to conduct, which validates its clinical significance and prognosis in patients with chondrosarcomas.

Although the Cancer Genome Atlas showed that ZNF704 expression in prostate cancer is lower as compared to benign prostate samples, ${ }^{8}$ suggesting a tumorsuppressive role, it can definitely exert as a potent tumor-promoting role in tumorigenesis in many reports. ${ }^{6,7}$ In addition, ZNF704 is an important regulator of the circadian clock and a potential driver for breast carcinogenesis. ${ }^{6}$ Consistent with these results, our results displayed that knockdown of ZNF704 can suppress chondrosarcoma cell viability, colony formation, and induce apoptosis in vitro and in vivo (Figure 3), supporting the evidence for its tumor-promoting role in chondrosarcoma development. Our findings, combination with the previous studies, ${ }^{6,7}$ suggest that ZNF704 may act as a oncogenic factor, potentiating chondrosarcoma cell growth linked to apoptosis. In fact, defects in apoptotic pathways are thought to contribute to the pathogenesis of human cancers, and may result in the survival of malignant tumors. ${ }^{13}$ Therefore, induction of apoptosis mediated by knockdown of ZNF704 in chondrosarcoma cells represents a novel treatment strategy targeting chondrosarcoma. However, the precise molecular mechanisms by which ZNF704 suppresses chondrosarcoma cell growth through induction of apoptosis certainly merit further investigations.

\section{Conclusions}

Our work identifies increased levels of ZNF704 significantly associated with higher grade chondrosarcoma, advanced stage, and a worse prognosis in patients with chondrosarcoma, supporting that ZNF704 acts as a potent oncogene in chondrosarcoma development. We have also discovered that ZNF704 is recognized as an independent prognostic factor and promotes the growth of chondrosarcoma in vitro and in vivo, highlighting a novel biomarker and attractive target for chondrosarcoma treatment.

\section{Data Sharing Statement}

The datasets used and/or analyzed during this study are available from the corresponding author on reasonable request.

\section{Ethics Approval and Consent to Participate}

Clinical tissues such as benign cartilage tumors and chondrosarcomas were handled in accordance with the Declaration of Helsinki and the Human Tissue Act. The sample collection in this study were obtained as discarded tissues after written informed consent was obtained from all patients, and the use of biological tissue material in the present project was covered by the ethical approvals from the Ethics Commission Peking University Third Hospital and Tianjin Hospital as described previously (IRB00001052-08044). ${ }^{10,11}$ The animal study was approved by the Animal Care and Use Committee and strictly followed ethical regulatory standards. ${ }^{12}$ Mice were housed and maintained in a pathogen-free facility, and all experimental procedures and protocols were approved by the Institutional Authority for Laboratory Animal Care, and also in accordance with guidelines for the ethical review of laboratory animal welfare People's Republic of China National Standard GB/T 35892-2018.

\section{Acknowledgments}

This work was supported by grants from the National Natural Sciences Foundation of China (81102037), and was also supported by grants from TianJin Youth Medicine Talents Plan.

\section{Author Contributions}

All authors made substantial contributions to conception and design, data analysis, drafting or revising the article, have agreed on the journal to which the article will be submitted, gave final approval of the version to be published, and agree to be accountable for all aspects of the work.

\section{Disclosure}

All authors declared that they have no competing interest in this work.

\section{References}

1. Amer KM, Munn M, Congiusta D, Abraham JA, Basu Mallick A. Survival and prognosis of chondrosarcoma subtypes: SEER database analysis. J Orthop Res. 2020;38(2):311-319. doi:10.1002/jor.24463 
2. Mery B, Espenel S, Guy JB, et al. Biological aspects of chondrosarcoma: leaps and hurdles. Crit Rev Oncol Hematol. 2018;126:32-36. doi:10.1016/j.critrevonc.2018.03.009

3. MacDonald IJ, Lin CY, Kuo SJ, Su CM, Tang CH. An update on current and future treatment options for chondrosarcoma. Expert Rev Anticancer Ther. 2019;19(9):773-786. doi:10.1080/14737140.2019.1659731

4. Ye Q, Liu J, Xie K. Zinc finger proteins and regulation of the hallmarks of cancer. Histol Histopathol. 2019;34(10):1097-1109. doi:10.14670/HH-18-121

5. Jen J, Wang YC. Zinc finger proteins in cancer progression. J Biomed Sci. 2016;23(1):53. doi:10.1186/s12929-016-0269-9

6. Yang C, Wu J, Liu X, et al. Circadian rhythm is disrupted by ZNF704 in breast carcinogenesis. Cancer Res. 2020;80(19):4114-4128. doi:10.1158/0008-5472.CAN-20-0493

7. Chen HQ, Zhao J, Li Y, et al. Gene expression network regulated by DNA methylation and microRNA during microcystin-leucine arginine induced malignant transformation in human hepatocyte L02 cells. Toxicol Lett. 2018;289:42-53. doi:10.1016/j.toxlet.2018.03.003

8. Cheng A, Zhao S, FitzGerald LM, et al. A four-gene transcript score to predict metastatic-lethal progression in men treated for localized prostate cancer: development and validation studies. Prostate. 2019;79(14):1589-1596. doi:10.1002/pros.23882
9. Chen C, Ma Q, Ma X, Liu Z, Liu X. Association of elevated HIF-2alpha levels with low Beclin 1 expression and poor prognosis in patients with chondrosarcoma. Ann Surg Oncol. 2011;18 (8):2364-2372. doi:10.1245/s10434-011-1587-5

10. Chen C, Zhou H, Zhang X, Liu Z, Ma X. PRMT1 potentiates chondrosarcoma development through activation of YAP activity. Mol Carcinog. 2019;58(12):2193-2206. doi:10.10 02/mc. 23108

11. Chen C, Tian A, Zhou H, Zhang X, Liu Z, Ma X. Upregulation of miR-211 promotes chondrosarcoma development via targeting tumor suppressor VHL. Onco Targets Ther. 2020;13: 2935-2943.

12. Chen C, Tian A, Zhao M, Ma X. Adenoviral delivery of VHL suppresses bone sarcoma cell growth through inhibition of Wnt/ beta-catenin signaling. Cancer Gene Ther. 2019;26(3-4):83-93. doi:10.1038/s41417-018-0041-2

13. Hanahan D, Weinberg RA. Hallmarks of cancer: the next generation. Cell. 2011;144:646-674. doi:10.1016/j.cell.2011.02.013

\section{Publish your work in this journal}

Cancer Management and Research is an international, peer-reviewed open access journal focusing on cancer research and the optimal use of preventative and integrated treatment interventions to achieve improved outcomes, enhanced survival and quality of life for the cancer patient.
The manuscript management system is completely online and includes a very quick and fair peer-review system, which is all easy to use. Visit http://www.dovepress.com/testimonials.php to read real quotes from published authors. 